\title{
CORRESPONDENCE
}

\section{Cross-reactive antibody immunity against SARS-CoV-2 in children and adults}

\author{
Elizabeth Fraley ${ }^{1}$, Cas LeMaster ${ }^{1}$, Dithi Banerjee ${ }^{2}$, Santosh Khanal ${ }^{1}$, Rangaraj Selvarangan ${ }^{2,4}$ and Todd Bradley ${ }^{1,3,4,5}$ \\ Cellular \& Molecular Immunology (2021) 18:1826-1828; https://doi.org/10.1038/s41423-021-00700-0
}

Severe acute respiratory syndrome coronavirus 2 (SARS-CoV-2) is a novel beta-coronavirus that has emerged and caused a global pandemic of coronavirus disease 2019 (COVID-19). ${ }^{1,2}$ Clinical manifestations of COVID-19 are age dependent, with children appearing to be less vulnerable than adults to severe disease. ${ }^{3,4}$ It has been reported that a subset of children and adults without previous infection had higher levels of humoral and cellular immunity to SARS-CoV-2, which may influence disease severity. ${ }^{5-8}$ Another study found that children who had never been exposed to SARS-CoV-2 had higher levels of cross-reactive antibodies to the spike glycoprotein subunit S2 of SARS-CoV-2 than adults. ${ }^{9}$ These findings raise the hypothesis that children may have a higher degree of preexisting cross-reactive immunity that provides some level of protection from severe COVID-19. In this study, we determined antibody responses to SARS-CoV-2 in adults after infection and compared these responses in both children and adults with no prior exposure to SARS-CoV-2. Moreover, we mapped antibody epitopes elicited after infection, and crossreactive antibody epitopes without prior SARS-CoV-2 infection, at high resolution using SARS-CoV-2 spike protein peptide arrays.

We determined lgG antibody responses using a multiplexed fluorescent bead assay that detects levels of antibodies against the SARS-CoV-2 nucleocapsid protein (NP) and three spike protein subunits (S1, S2, and receptor-binding domain (RBD)). First, we used peripheral blood from 44 adults of diverse age, sex, and race/ ethnicity who had a positive SARS-CoV-2 PCR test 2-133 days prior to blood sampling (Table S1). None of the individuals experienced severe COVID-19 that required hospitalization. We defined a detection threshold of $230 \mathrm{MFI}$ (sum of the average and standard deviation of the highest negative control beads without antigen). All 44 individuals had detectable lgG antibody responses to the S2 and RBD proteins; 38 and 41 of the individuals also had responses to the NP and S1 proteins, respectively (Fig. 1A). It has been reported that a subset of children and adults without previous exposure had higher levels of humoral and cellular immunity to SARS-CoV-2, which may influence disease severity. 5,6 Therefore, we also determined IgG antibody responses to SARS-CoV-2 in individuals not previously exposed to SARS-CoV-2 using 44 healthy adults recruited in early 2019 and 86 children recruited in 2016 and 2017 prior to the COVID19 outbreak (Table S2). Forty-one of the 44 adults and 57 of the 86 children without prior SARS-CoV-2 infection had detectable lgG antibodies against the SARS-CoV-2 spike S2 subunit; 41 and 46 adults and children had detectable antibodies targeting NP (Fig. 1A).
Preexisting immunity to the S1 antigen was rarely detectable ( 1 adult and 3 children; Fig. 1A). Levels of SARS-CoV-2 antibodies in adults and children without prior infection were, on average, an order of magnitude lower than those soon after natural infection.

We created a more rigorous cutoff for positivity by averaging the MFI values for SARS-CoV-2-negative adults and children plus the standard deviation for each viral antigen to compare differences in the frequency of preexisting antibodies between age groups. Using this cutoff, we found that uninfected infants (less than 1-year-old) did not have cross-reactive SARS-CoV-2 antibodies, with only 1 of 19 infants having a positive IgG-RBD antibody titer and no positive response to any other SARS-CoV-2 viral antigens (Fig. S1). Adults showed a positivity rate between $13.6 \%$ and $18.3 \%$ for the S2, RBD, and NP antigens, whereas children (ages 2-10 and 11-15) had similar rates for S2 and RBD (averaged $16 \%$ and 12.8\%) but a lower frequency of IgG-NP antibody positivity (averaged 3.2\%; Fig. S1). Thus, children and adults without COVID-19 had detectable IgG crossreactive antibodies to SARS-CoV-2 viral antigens, with the highest responses to $\mathrm{S} 2, \mathrm{RBD}$, and NP and rare responses to the $\mathrm{S} 1$ subunit. Interestingly, infants aged $0-1$ years old did not have detectable cross-reactive antibodies, and only subtle differences in positive responses between uninfected adults and children aged 2-15 years old were observed. Although detectable, the magnitude of these responses was lower than that observed soon after natural infection.

We selected 14 SARS-CoV-2-infected individuals at an average of 25 days after infection (seropositive), seven seronegative children (C) and seven seronegative adults (A) and used peptide microarrays containing 196 soluble 12-mer overlapping peptides that fully cover the SARS-CoV-2 spike protein for high-resolution mapping of the antibody response after infection (Supplementary Data 1, Supplementary Data 2 and Supplementary Data 3). For the spike peptide array, immunodominant regions in the N-terminal domain and RBD of the S1 subunit were detected for both seropositive and seronegative individuals (Fig. 1B). Regarding the S2 subunit, immunodominant regions near the fusion peptide, HR1 and C terminus were recognized by antibodies from both seropositive and seronegative individuals (Fig. 1C). Moreover, three immunodominant peptides (S1-61, S1-66, and S1-76) in the RBD were present in both seropositive and seronegative individuals (Fig. 1B). The sequences of these peptides are conserved among closely related and emergent group B coronaviruses such as SARS-CoV-1 but not group $C$ beta-coronaviruses such as Middle Eastern Respiratory Syndrome (MERS) (Fig. S2A).

${ }^{1}$ Genomic Medicine Center, Children's Mercy Research Institute, Children's Mercy Kansas City, Kansas City, MO, USA; ${ }^{2}$ Department of Pathology and Laboratory Medicine, Children's Mercy Research Institute, Children's Mercy Kansas City, Kansas City, MO, USA; ${ }^{3}$ Department of Pediatrics, Children's Mercy Kansas City, Kansas City, MO, USA; ${ }^{4}$ Department of Pediatrics, UMKC School of Medicine, Kansas City, MO, USA and ${ }^{5}$ Departments of Pediatrics and Pathology and Laboratory Medicine, University of Kansas Medical Center, Kansas City, KS, USA

Correspondence: Rangaraj Selvarangan (rselvarangan@cmh.edu) or Todd Bradley (tcbradley@cmh.edu)

Received: 5 May 2021 Accepted: 10 May 2021

Published online: 31 May 2021 


\section{A Adults (Prior Infection)}

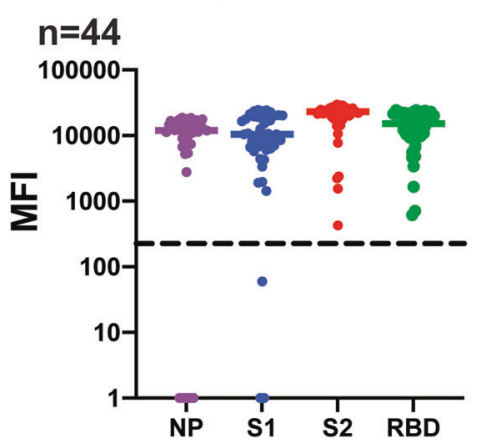

B
Adults (no infection)

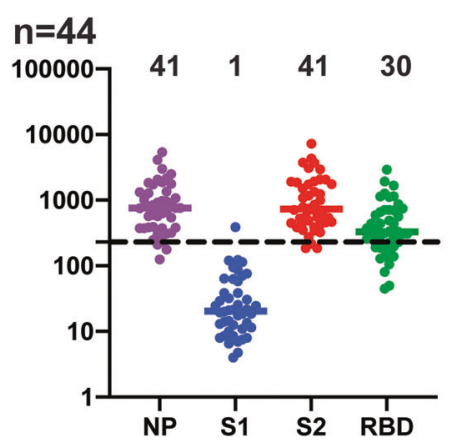

SARS-CoV-2 S1 peptide array
RBD

SARS-CoV-2 S1 peptide array
RBD

\section{Children (no infection)}

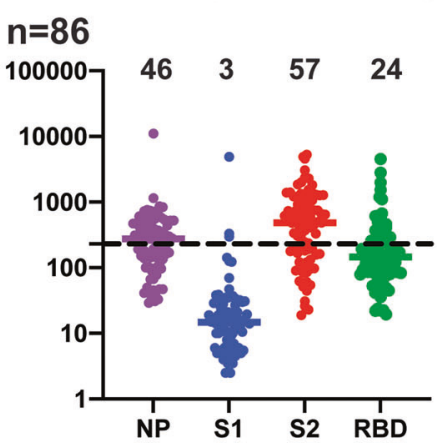

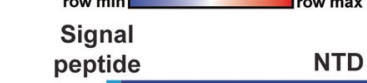

vage site
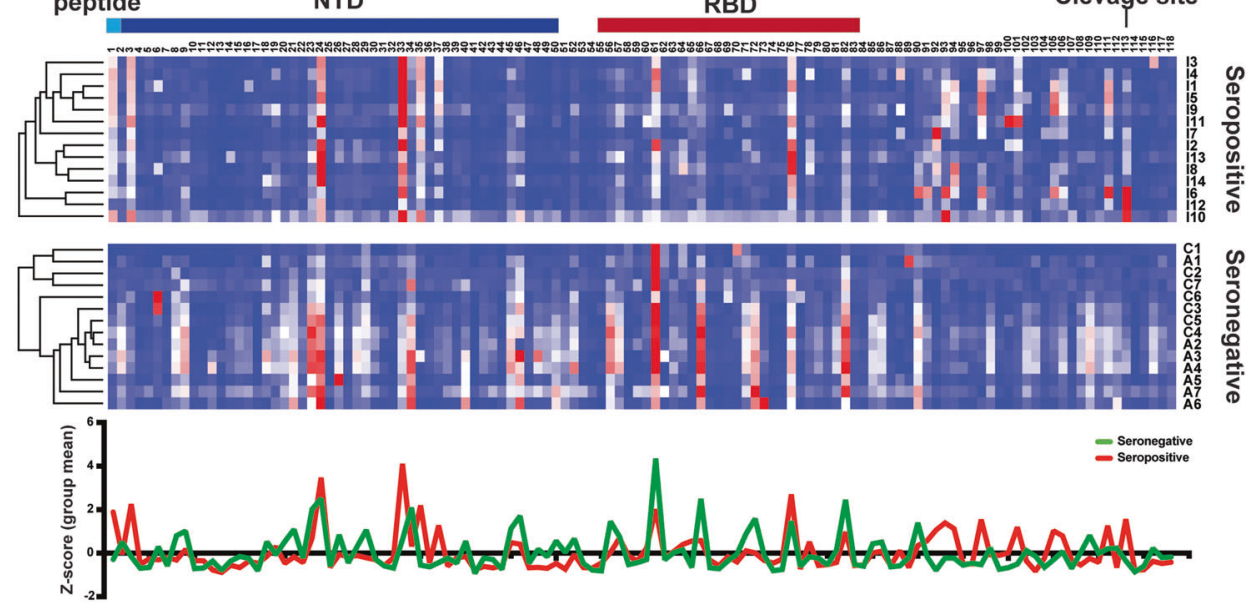

C
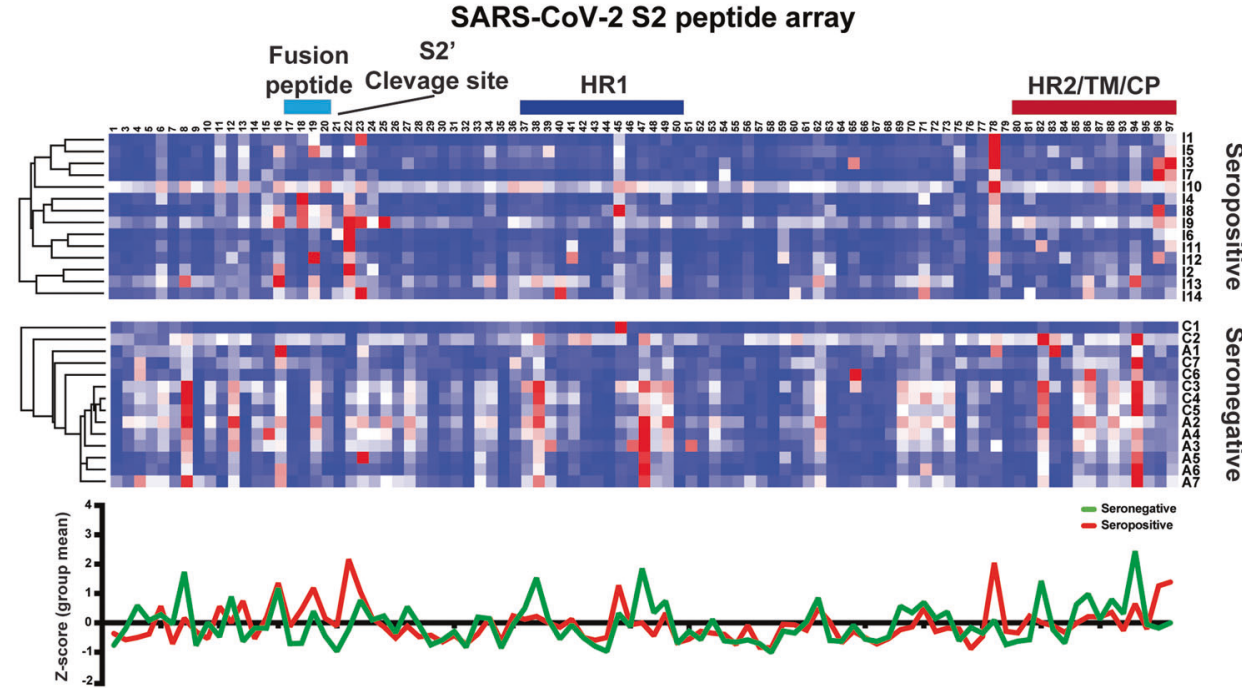

Fig. 1 Antibody responses to SARS-CoV-2 in previously infected and healthy populations. (A) Multiplex bead-based antibody binding assay to measure the IgG antibody response to four SARS-CoV-2 viral antigens (S1, S2, RBD, and NP). The median fluorescence intensity (MFI) is shown, and background well subtraction was used to remove nonspecific signals. Each dot represents an individual in three groups: SARSCoV-2 convalescent adults (left, $n=44$ ), adults with no prior SARS-CoV-2 infection (middle, $n=44$ ), and pediatric individuals with no prior SARS-CoV-2 (right, $n=866$ ). Bars represent the group median. The dashed line indicates a threshold determined by the sum of the mean and standard deviation for the negative control (beads without antigen). The numbers above each antigen are the numbers of individuals above the determined threshold. Plasma IgG binding to SARS-CoV-2 peptides spanning the SARS-CoV-2 spike protein ((B) S1 and (C) S2 subunits; $12-$ mer overlapping peptides). Seropositive (top) represents 14 individuals previously infected with SARS-CoV-2 (I1-I14), and seronegative (bottom) represents seven children (C1-C7) and seven adults (A1-A7) recruited prior to the COVID-19 pandemic. Each peptide was printed in triplicate, and the Log2 of the mean fluorescent intensity (F635) for each peptide was graphed. The row color corresponds to the minimum and maximum intensity for all peptides for each individual. Known regions of the spike protein are annotated above the heatmaps. Line graphs display the mean group Z-score for each peptide in S1 and S2 from the seronegative group (green; $n=14$ ) and seropositive group (red; $n=14)$ 
In addition, we identified 11 peptides with significantly higher binding in the seropositive group compared to the seronegative group, and 28 peptides with significantly higher binding in the seronegative group compared to the seropositive group, based on $Z$-score values for each array peptide that identified differences in the antibody epitope response during SARS-CoV-2 infection compared to preexisting cross-reactive immunity in seronegative individuals (Fig. S2B; Table S3; $t$-test, adjusted $p$ value $\leq 0.05$ ). These unique peptides could represent epitopes specific to SARSCoV-2 and other coronaviruses. These results identified regions in the spike protein that are commonly recognized by antibodies from many SARS-CoV-2-infected or SARS-CoV-2 cross-reactive antibodies detected in seronegative individuals and could serve as a guide for future vaccine design.

A detailed understanding of humoral immunity after SARS-CoV2 infection and the breadth of preexisting cross-reactive immunity will enable better treatment, diagnostic and vaccine strategies, which will aid in controlling the current and future pandemics. We determined that children and adults without SARS-CoV-2 infection history had preexisting cross-reactive humoral immunity that may contribute to disease pathogenesis. However, cross-reactive humoral immunity to SARS-CoV-2 is only one aspect of the immune system that may play a protective role in preventing severe COVID-19. Indeed, it has also been demonstrated that high frequencies of uninfected individuals mount preexisting crossreactive $T$ cell immune responses to SARS-CoV-2. ${ }^{5,6,10}$ The identification of these broad $B$ and $T$ cell epitopes with protective efficacy might serve as future targets for pancoronavirus vaccines and also predict disease severity after coronavirus infection.

\section{ACKNOWLEDGEMENTS}

We thank Bradley Beldon for clinical coordination and Elin Grundberg for scientific discussion and suggestions during this work. This work was funded through internal institutional funds from Children's Mercy Research Institute and Children's Mercy Kansas City.

\section{AUTHOR CONTRIBUTIONS}

Conceptualization: R.S., T.B.; sample acquisition: E.F., C.L., D.B., R.S., T.B.; laboratory analysis: C.L., E.F., D.B.; data analysis: C.L., E.F., S.K., D.B., T.B.; writing: E.F., T.B.; editing: all authors; funding: T.B., R.S.

\section{ADDITIONAL INFORMATION}

Supplementary information The online version contains supplementary material available at https://doi.org/10.1038/s41423-021-00700-0.

Competing interests: The authors declare no competing interests.

\section{REFERENCES}

1. Wu, F. et al. A new coronavirus associated with human respiratory disease in China. Nature 579, 265-269 (2020).

2. Zhu, N. et al. A Novel Coronavirus from Patients with Pneumonia in China, 2019. N. Engl. J. Med 382, 727-733 (2020).

3. Guan, W. J. et al. Clinical Characteristics of Coronavirus Disease 2019 in China. N. Engl. J. Med 382, 1708-1720 (2020).

4. Dong, Y. et al. Epidemiology of COVID-19 Among Children in China. Pediatrics 145, https://doi.org/10.1542/peds.2020-0702 (2020).

5. Mateus, J. et al. Selective and cross-reactive SARS-CoV-2 T cell epitopes in unexposed humans. Science 370, 89-94 (2020).

6. Shrock, E. et al. Viral epitope profiling of COVID-19 patients reveals crossreactivity and correlates of severity. Science 370, https://doi.org/10.1126/science. abd4250 (2020).

7. Selva, K. J. et al. Systems serology detects functionally distinct coronavirus antibody features in children and elderly. Nat. Commun. 12, 2037 (2021).

8. Guo, L. et al. Cross-reactive antibody against human coronavirus OC43 spike protein correlates with disease severity in COVID-19 patients: a retrospective study. Emerg. Microbes Infect. 10, 664-676 (2021).

9. $\mathrm{Ng}, \mathrm{K}$. W. et al. Preexisting and de novo humoral immunity to SARS-CoV-2 in humans. Science 370, 1339-1343 (2020).

10. Grifoni, A. et al. Targets of T Cell Responses to SARS-CoV-2 Coronavirus in Humans with COVID-19 Disease and Unexposed Individuals. Cell 181, 1489-1501. e1415 (2020). 